\title{
EXPLICIT EVALUATION OF SOME OF THE THETA-FUNCTION IDENTITIES
}

\author{
SHRUTHI AND B. R. SRIVATSA KUMAR
}

Received 24 October, 2019

\begin{abstract}
In this paper, we evaluate two parameters $h_{k, n}$ and $h_{k, n}^{\prime}$ of some $P-Q$ type Theta functions $\varphi(q)$ for any positive real numbers $k$ and $n$. During this process, we also evaluate Ramanujan's cubic continued fraction.
\end{abstract}

2010 Mathematics Subject Classification: 33D10; 11F27

Keywords: Theta function, Modular equation, Ramanujan's cubic continued fraction

\section{INTRODUCTION}

For any complex number $z, q=e^{2 \pi i z}, \operatorname{Im}(z)>0$, define

$$
\varphi(q):=\sum_{n=-\infty}^{\infty} q^{n^{2}}=: \Theta_{3}(0,2 z)
$$

and

$$
f(-q):=(q ; q)_{\infty}=q^{-1 / 24} \eta(z),
$$

where $\Theta_{3}$ is the classical theta-function [23, p. 464] and $\eta(z)$ denotes the Dedekind eta-function and $(a ; q)_{\infty}$ is defined by

$$
(a ; q)_{\infty}:=\prod_{k=0}^{\infty}\left(1-a q^{k}\right) .
$$

It is precisely assumed in the sequel that $|q|<1$ always. Recently, J. Yi [24] evaluated many new values of $\varphi(q)$ using modular identities, transformation formula for thetafunctions and are defined as follows:

Definition 1. For any positive real number $k$ and $n$ we have

$$
h_{k, n}:=\frac{\varphi(q)}{k^{1 / 4} \varphi\left(q^{k}\right)}=\frac{\Theta_{3}(0, i \sqrt{n / k})}{k^{1 / 4} \Theta_{3}(0, i \sqrt{n k})} \quad q=e^{-\pi \sqrt{n / k}},
$$

The second author thank SERB, DST, India for sanctioning the project [EMR/2016/001601]. 


$$
h_{k, n}^{\prime}:=\frac{\varphi(-q)}{k^{1 / 4} \varphi\left(-q^{k}\right)}=\frac{\Theta_{3}(0,1+2 i \sqrt{n / k})}{k^{1 / 4} \Theta_{3}(0,1+2 i \sqrt{n k})} \quad q=e^{-2 \pi \sqrt{n / k}} .
$$

Also it is observed that

$$
\begin{array}{cl}
\text { i. } & h_{k, 1}=1, \\
\text { ii. } & h_{k, 1 / n}=h_{k, n}^{-1}, \\
\text { iii. } & h_{k, n}=h_{n, k} .
\end{array}
$$

The Ramanujan-Göllnitz-Gordan continued fraction $H(q)$ is defined as

$$
H(q):=\frac{q^{1 / 2}}{1+q_{+}} \frac{q^{2}}{1+q^{3}}+\frac{q^{4}}{1+q^{5}}+\ldots
$$

The above continued fraction was introduced by S. Ramanujan in his second notebook [16, p. 229]. H. Göllnitz [11] and B. Gordon [12] rediscovered $H(q)$ without knowing of Ramanujan's work. Ramanujan also recorded following two identities for $H(q)$ in his second notebook [16, p. 229],

$$
\frac{1}{H(q)}-H(q)=\frac{\varphi\left(q^{2}\right)}{q^{1 / 2} \psi\left(q^{4}\right)} \quad \text { and } \quad \frac{1}{H(q)}+H(q)=\frac{\varphi(q)}{q^{1 / 2} \psi\left(q^{4}\right)} .
$$

Proofs of the above two identities can be found in [4, p. 221]. H. H. Chan and S. S. Haung [10], have established many identities for $H(q)$, which are analogues to the results of famous Roger-Ramanujan continued fraction and Ramanujan's cubic continued fraction. Chan and Haung [10] have also derived some explicit formulas for evaluating $H\left(e^{-\pi \sqrt{n} / 2}\right)$ in terms of Ramanujan-Weber class invariants. Recently C. Adiga et. al. [2] have established several modular relations for the RogersRamanujan type functions of order eleven which are analogues to Ramanujan's forty identities and also they established certain interesting partition theoretic interpretations. H. M. Srivastava and M. P. Chaudhary [17] established a set of four new results which depict the interrelationships between $q$-identities, continued fraction identities and combinatorial partition identities. Also in $[18,19]$ H. M. Srivastava et. al. deduced some $q$-identities involving theta functions. These $q$-identities have relationship among three of the theta-type functions which arise from Jacobi's triple product identity. In [22], K. R. Vasuki and B. R. Srivatsa Kumar proved the following:

Lemma 1. For $q=e^{-\pi \sqrt{k / 2}}$, let

$$
J_{k}:=\sqrt{2} \frac{\varphi^{2}\left(q^{2}\right)}{\varphi^{2}(q)},
$$

then

$$
\begin{array}{rc}
\text { i. } & J_{k} J_{1 / k}=1, \\
\text { ii. } & J_{1}=1, \\
\text { iii. } & H(q)=\sqrt{\frac{\sqrt[4]{2}-\sqrt{J_{k}}}{\sqrt[4]{2}+\sqrt{J_{k}}} .}
\end{array}
$$


In his first notebook [16] Ramanujan recorded many elementary values of $\varphi(q)$. Particularly, he recorded $\varphi\left(e^{-n \pi}\right)$ and $\varphi\left(-e^{-n \pi}\right)$ for $n=1,2,4,8,1 / 2$ and $1 / 4$. All these values are proved by Berndt [5, p. 325]. Ramanujan also recorded non-elementary values of $\varphi\left(e^{-n \pi}\right)$ for $n=3,5,7,9$ and 45. And all these are proved by Berndt and Chan [7] . Recently Yi [24], evaluated $\varphi\left(e^{-n \pi}\right)$ for $n=1,2,3,4,5$ and 6 and $\varphi\left(-e^{n \pi}\right)$ for $n=1,2,4,6,8,10$ and 12. Furthermore, M. S. M. Naika and Chandan kumar [13] and Naika et. al [14] established several general formulas for the explicit evaluation of $h_{2, n}$ and $h_{4, n}$ by employing modular equation of degree 2 and 4 respectively. On page 366 of his lost notebook [15, p. 248], Ramanujan recorded another continued fraction

$$
G(q):=\frac{q^{1 / 3}}{1}+\frac{q+q^{2}}{1}+\frac{q^{2}+q^{4}}{1}+\frac{q^{3}+q^{6}}{1}+\ldots .
$$

and claimed that there are many results of $G(q)$. Motivated by Ramanujan's claim, H. H. Chan [9] derived many new identities which Ramanujan vaguely referred. Recently B. C. Berndt, Chan and L. -C. Zhang [6], N. D. Baruah and Nipen Saikia [3], C. Adiga et. al. [1], S. Bhargava et. al. [8] have found several explicit values of $G(q)$. Further, as a particular case of this for $k=3$ they proved the following:

Lemma 2. For $q=e^{-\pi \sqrt{n / 3}}$, let

$$
J_{n}:=\frac{1}{\sqrt[4]{3}} \frac{\varphi(q)}{\varphi\left(q^{3}\right)},
$$

then

$$
\begin{array}{cl}
\text { i. } & J_{n} J_{1 / n}=1, \\
\text { ii. } & J_{1}=1, \\
\text { iii. } & D_{n}=\sqrt{\frac{\sqrt{3}-J_{n}^{2}}{1+\sqrt{3} J_{n}^{2}},} \\
\text { iv. } & G(q)=\frac{1}{2} \sqrt[3]{1-3 D_{n}^{4}} .
\end{array}
$$

Motivated by the above work, in this paper we find some general formulas for the explicit evaluation of $h_{2, n}, h_{3, n}$ and $h_{3, n}^{\prime}$. Also we evaluate Ramanujan's cubic continued fraction and Ramanujan-Göllintz-Gordon continued fraction.

\section{PReliminary RESUlts: $P-Q$ TyPe Theta-FUnCTION IDENTITIES}

In this section, we state $P-Q$ type theta-function identities and also some $h_{k, n}$ and $h_{k, n}^{\prime}$ which we need in sequel.

$$
\begin{gathered}
\text { Theorem } 1 \text { ([8]). If } P:=\frac{\varphi(q)}{\varphi\left(q^{3}\right)} \quad \text { and } Q:=\frac{\varphi(-q)}{\varphi\left(-q^{3}\right)} \text { then } \\
\frac{P}{Q}+\frac{Q}{P}=\frac{3}{P Q}-P Q .
\end{gathered}
$$


Theorem 2 ([8]). If $P:=\frac{\varphi(-q)}{\varphi\left(-q^{3}\right)} \quad$ and $\quad Q:=\frac{\varphi\left(-q^{2}\right)}{\varphi\left(-q^{6}\right)}$ then

$$
\left(\frac{P}{Q}\right)^{2}+\left(\frac{Q}{P}\right)^{2}=\frac{3}{Q^{2}}-Q^{2}
$$

Theorem 3 ([8]). If $P:=\frac{\varphi(q)}{\varphi\left(q^{3}\right)} \quad$ and $\quad Q:=\frac{\varphi\left(q^{2}\right)}{\varphi\left(q^{6}\right)}$ then

$$
(P Q)^{2}+\frac{9}{(P Q)^{2}}+2\left(P^{2}-Q^{2}\right)=6\left(\frac{1}{P^{2}}-\frac{1}{Q^{2}}\right)-\left(\frac{P}{Q}\right)^{2}-\left(\frac{Q}{P}\right)^{2}+12 .
$$

Theorem 4 ([22]). If $P:=\frac{\varphi^{2}\left(q^{2}\right)}{\varphi^{2}(q)} \quad$ and $\quad Q:=\frac{\varphi^{2}\left(q^{4}\right)}{\varphi^{2}\left(q^{2}\right)}$ then

$$
4 P^{2} Q^{2}-4 P^{2} Q+P^{2}-2 P+1=0 .
$$

Theorem 5 ([22]). If $P:=\frac{\varphi^{2}\left(q^{2}\right)}{\varphi^{2}(q)} \quad$ and $\quad Q:=\frac{\varphi^{2}\left(q^{6}\right)}{\varphi^{2}\left(q^{3}\right)}$ then $64 P Q+\frac{16}{P Q}-96(P+Q)-48\left(\frac{1}{P}+\frac{1}{Q}\right)+138=\left(\frac{Q}{P}\right)^{2}+\left(\frac{P}{Q}\right)^{2}-36\left(\frac{P}{Q}+\frac{Q}{P}\right)$.

Theorem 6 ([20]). If $P:=\frac{\varphi(q)}{\varphi\left(q^{3}\right)} \quad$ and $\quad Q:=\frac{\varphi\left(q^{7}\right)}{\varphi\left(q^{21}\right)}$ then

$$
\begin{aligned}
\left(\frac{P}{Q}\right)^{4}-\left(\frac{Q}{P}\right)^{4} & +14\left(\left(\frac{P}{Q}\right)^{2}-\left(\frac{Q}{P}\right)^{2}\right)-7\left(\left(\frac{P}{Q}\right)^{2}+\left(\frac{Q}{P}\right)^{2}-1\right) \\
& \times\left(P Q+\frac{3}{P Q}\right)+(P Q)^{3}+\frac{27}{(P Q)^{3}}=0 .
\end{aligned}
$$

Theorem 7 ([20]). If $\quad P:=\frac{\varphi\left(q^{3}\right) \varphi\left(q^{2}\right)}{\varphi(q) \varphi\left(q^{6}\right)} \quad$ and $\quad Q:=\frac{\varphi\left(q^{6}\right) \varphi\left(q^{4}\right)}{\varphi\left(q^{2}\right) \varphi\left(q^{12}\right)} \quad$ then $(P Q)^{2}+\frac{1}{(P Q)^{2}}-8\left(P Q+\frac{1}{P Q}\right)+6\left(\sqrt{P Q}-\frac{1}{\sqrt{P Q}}\right)\left(\sqrt{\frac{P}{Q}}+\sqrt{\frac{Q}{P}}\right)$
$-2\left((P Q)^{3 / 2}-\frac{1}{(P Q)^{3 / 2}}\right)\left(\sqrt{\frac{P}{Q}}+\sqrt{\frac{Q}{P}}\right)+\left(P Q+\frac{1}{P Q}\right)\left(\frac{P}{Q}+\frac{Q}{P}\right)+10=0$.

Theorem 8 ([20]). If $P:=\frac{\varphi^{2}\left(q^{3}\right)}{\varphi(q) \varphi\left(q^{9}\right)} \quad$ and $\quad Q:=\frac{\varphi^{2}\left(q^{6}\right)}{\varphi\left(q^{2}\right) \varphi\left(q^{18}\right)} \quad$ then

$$
\left(\frac{P}{Q}\right)^{2}+\left(\frac{Q}{P}\right)^{2}-2\left(P Q-\frac{3}{P Q}\right)\left(\frac{P}{Q}+\frac{Q}{P}\right)+(P Q)^{2}+\frac{9}{(P Q)^{2}}
$$




$$
-16\left(P Q+\frac{3}{P Q}\right)-44=0 .
$$

Theorem 9 ([20]). If $\quad P:=\frac{\varphi\left(q^{3}\right) \varphi\left(q^{5}\right)}{\varphi(q) \varphi\left(q^{15}\right)} \quad$ and $\quad Q:=\frac{\varphi\left(q^{6}\right) \varphi\left(q^{10}\right)}{\varphi\left(q^{2}\right) \varphi\left(q^{30}\right)}$ then

$$
\begin{aligned}
& (P Q)^{2}+\frac{1}{(P Q)^{2}}-24\left(P Q+\frac{1}{P Q}\right)+\left(\frac{P}{Q}\right)^{2}+\left(\frac{Q}{P}\right)^{2}+\left[6\left(P Q+\frac{1}{P Q}\right)-8\right] \\
& \times\left(\frac{P}{Q}+\frac{Q}{P}\right)-4\left(\sqrt{P Q}-\frac{1}{\sqrt{P Q}}\right)\left(\left(\frac{P}{Q}\right)^{3 / 2}+\left(\frac{Q}{P}\right)^{3 / 2}\right) \\
& +\left[16\left(\sqrt{P Q}-\frac{1}{\sqrt{P Q}}\right)-4\left((P Q)^{3 / 2}-\frac{1}{(P Q)^{3 / 2}}\right)\right]\left(\sqrt{\frac{P}{Q}}+\sqrt{\frac{Q}{P}}\right)+36=0
\end{aligned}
$$

Lemma 3 ([24]). If $h_{k, n}$ and $h_{k, n}^{\prime}$ are as defined as in (1.1) and (1.2) then, we have

$$
\begin{aligned}
& h_{2,4}=\sqrt{2}+1-\sqrt{\sqrt{2}+1}, \quad h_{2,8}=\frac{\sqrt{2+\sqrt{2}}}{\sqrt[4]{2}+1}, \quad h_{3,3}=(2 \sqrt{3}-3)^{1 / 4}=\frac{3^{1 / 8} \sqrt{\sqrt{3}-1}}{2^{1 / 4}}, \\
& h_{3,1 / 3}=\left(\frac{2 \sqrt{3}+3}{3}\right)^{1 / 4}=\frac{\sqrt{\sqrt{3}+1}}{2^{1 / 4} 3^{1 / 8}}, \quad h_{3,5}=\frac{\sqrt{\sqrt{5}-1}}{\sqrt{2}}, \quad h_{3,1 / 5}=\frac{\sqrt{\sqrt{5}+1}}{\sqrt{2}}, \\
& h_{3,9}=\frac{1}{\sqrt{3}}(1-\sqrt[3]{2}+\sqrt[3]{4}), \quad h_{3,1 / 9}=\frac{1+\sqrt[3]{2}}{\sqrt{3}}, \quad h_{3,1}^{\prime}=2^{-1 / 4} \sqrt{\sqrt{3}-1} .
\end{aligned}
$$

3. Evaluation OF $h_{k, n}$ AND $h_{k, n}^{\prime}$

Theorem 10. We have

$$
\begin{array}{ll}
\text { i. } & h_{3,6}=\sqrt[4]{603-426 \sqrt{2}-348 \sqrt{3}+246 \sqrt{6}}=h_{3,1 / 6}^{-1} \\
\text { ii. } & h_{3,2 / 3}=\frac{1}{3} \sqrt[4]{603-426 \sqrt{2}-348 \sqrt{3}+246 \sqrt{6}} \sqrt{9+6 \sqrt{2}}=h_{3,3 / 2}^{-1} .
\end{array}
$$

Proof. On using the definition of $h_{k, n}$ in Theorem 8 and by setting $n=1 / 6$, we deduce

$$
x^{2}+\frac{9}{x^{2}}-16\left(x+\frac{3}{x}\right)-4\left(x-\frac{3}{x}\right)+46=0
$$

where $x=\left(\frac{h_{3,6}}{h_{3,2 / 3}}\right)^{2}$. Now set $\frac{x}{\sqrt{3}}+\frac{\sqrt{3}}{x}=t, \frac{x^{2}}{3}+\frac{3}{x^{2}}=t^{2}-2$ and $\frac{x}{\sqrt{3}}-\frac{\sqrt{3}}{x}=\sqrt{t^{2}-4}$ in the above, we obtain

$$
9 t^{4}-96 \sqrt{3} t^{3}+960 t^{2}-1280 \sqrt{3} t+1792=0 .
$$


Since $h_{k, n}$ is decreasing, we choose $t=\frac{4}{\sqrt{3}}(3-\sqrt{2})$. Again on solving and since $\frac{h_{3,6}}{h_{3,2 / 3}}<1$, we have

$$
\frac{h_{3,6}}{h_{3,2 / 3}}=\sqrt{9-6 \sqrt{2}} \text {. }
$$

From [8, Theorem 3.4], if $P:=\frac{\varphi^{2}(q)}{\varphi^{2}\left(q^{3}\right)} \quad$ and $\quad Q:=\frac{\varphi^{2}\left(q^{3}\right)}{\varphi^{2}\left(q^{9}\right)}$ then

$$
P Q+\frac{9}{P Q}=3+6 \frac{Q}{P}+\frac{Q^{2}}{P^{2}} .
$$

On employing the definition of $h_{k, n}$ in the above, setting $n=1 / 6$ and using (3.1), we deduce

$$
y^{2}-(70-48 \sqrt{2}) y+1=0,
$$

where $y=\left(h_{3,6} h_{3,2 / 3}\right)^{2}$. On solving, we choose

$$
h_{3,6} h_{3,2 / 3}=\sqrt{35-24 \sqrt{2}-20 \sqrt{3}+14 \sqrt{6}} .
$$

From (3.1) and the above, we obtain the desired result.

Corollary 1. We have
i. $\quad D_{6}=\sqrt{\frac{\sqrt{3}-a}{1+\sqrt{3} a}}$,
$D_{1 / 6}=\sqrt{\frac{\sqrt{3} a-1}{a+\sqrt{3}}}$
ii. $\quad D_{2 / 3}=\frac{1}{\sqrt{3}} \sqrt{3-\sqrt{6}}$,
$D_{3 / 2}=\sqrt{\frac{\sqrt{3} b^{2}-1}{b^{2}+\sqrt{3}}}$

where

$$
a=\sqrt{603-426 \sqrt{2}-348 \sqrt{3}+246 \sqrt{6}} \text { and } b=\frac{1}{3} \sqrt{a(9+6 \sqrt{2})} .
$$

Proof. On using Theorem 10 to Lemma 2(iii), we obtain the above results.

Corollary 2. We have
i. $\quad G\left(e^{-\sqrt{2} \pi}\right)=\frac{1}{2} \sqrt[3]{1-3 D_{6}^{4}}$
$G\left(e^{-\pi / 3 \sqrt{2}}\right)=\frac{1}{2} \sqrt[3]{1-3 D_{1 / 6}^{4}}$
ii. $\quad G\left(e^{-\sqrt{2} \pi / 3}\right)=\frac{1}{2} \sqrt[3]{1-3 D_{2 / 3}^{4}}$,
$G\left(e^{-\pi / \sqrt{2}}\right)=\frac{1}{2} \sqrt[3]{1-3 D_{3 / 2}^{4}}$.

Proof. To prove the above results, we need to apply Corollary 1 to Lemma 2(iv) respectively.

Theorem 11. We have

$$
\text { i. } \quad h_{3,10}=\sqrt{(1+\sqrt{2})(2-\sqrt{3})} \sqrt[4]{99-40 \sqrt{6}+44 \sqrt{5}-18 \sqrt{30}}=h_{3,1 / 10}^{-1},
$$


ii. $\quad h_{3,2 / 5}=\frac{\sqrt[4]{99-40 \sqrt{6}+44 \sqrt{5}-18 \sqrt{30}}}{\sqrt{(1+\sqrt{2})(2-\sqrt{3})}}=h_{3,5 / 2}^{-1}$.

Proof. On using the definition of $h_{k, n}$ in Theorem 9 and by setting $n=1 / 10$, we deduce

$$
x^{4}+\frac{1}{x^{4}}-8\left(x^{3}-\frac{1}{x^{3}}\right)-12\left(x^{2}+\frac{1}{x^{2}}\right)+24\left(x-\frac{1}{x}\right)+22=0
$$

where $x=\frac{h_{3,10}}{h_{3,2 / 5}}$. Setting $x-x^{-1}=t$ in the above, we obtain $t^{2}\left(t^{2}-8 t-8\right)=0$. Since $h_{k, n}$ is positive and decreasing, we have $x-\frac{1}{x}=4-2 \sqrt{6}$. On solving this, we deduce

$$
\frac{h_{3,10}}{h_{3,2 / 5}}=(1+\sqrt{2})(2-\sqrt{3}) .
$$

From [21, Theorem 3.1], if $P:=\frac{\varphi(q)}{\varphi\left(q^{3}\right)} \quad$ and $\quad Q:=\frac{\varphi\left(q^{5}\right)}{\varphi\left(q^{15}\right)}$ then

$$
(P Q)^{2}+\frac{9}{(P Q)^{2}}=\left(\frac{Q}{P}\right)^{3}-\left(\frac{P}{Q}\right)^{3}+5\left(\left(\frac{Q}{P}\right)^{2}-\left(\frac{P}{Q}\right)^{2}\right)+5\left(\frac{Q}{P}-\frac{P}{Q}\right) .
$$

Again using the definition of $h_{k, n}$ in the above, by setting $n=2 / 5$ and then using (3.2), we deduce

$$
x^{2}+\frac{1}{x^{2}}=198-80 \sqrt{6}
$$

where $x=\left(h_{3,2 / 5} h_{3,10}\right)^{2}$. On solving this, we obtain

$$
h_{3,2 / 5} h_{3,10}=\sqrt{99-40 \sqrt{6}+44 \sqrt{5}-18 \sqrt{30}} .
$$

On using (3.2) and the above, we obtain the desired result.

Corollary 3. We have

$$
\begin{aligned}
\text { i. } & D_{10}=\sqrt{\frac{\sqrt{3}-a b}{1+\sqrt{3} a b},} & D_{1 / 10}=\sqrt{\frac{\sqrt{3} a b-1}{a b+\sqrt{3}}}, \\
\text { ii. } & D_{2 / 5}=\sqrt{\frac{1+\sqrt{2}-a-2 b}{b-\sqrt{3} a}}, & D_{5 / 2}=\sqrt{\frac{\sqrt{3} a-b}{a+\sqrt{3} b},}
\end{aligned}
$$

where

$$
a=\sqrt{99-40 \sqrt{6}+44 \sqrt{5}-18 \sqrt{30}} \quad \text { and } \quad b=2-\sqrt{3}+2 \sqrt{2}-\sqrt{6} .
$$

Proof. On using Theorem 11 to Lemma 2(iii), we obtain the above results. 
Corollary 4. We have
i.
$G\left(e^{-\pi \sqrt{10 / 3}}\right)=\frac{1}{2} \sqrt[3]{1-3 D_{10}^{4}}$
$G\left(e^{-\pi \sqrt{1 / 30}}\right)=\frac{1}{2} \sqrt[3]{1-3 D_{1 / 10}^{4}}$,
ii. $\quad G\left(e^{-\pi \sqrt{2 / 15}}\right)=\frac{1}{2} \sqrt[3]{1-3 D_{2 / 5}^{4}}$,
$G\left(e^{-\pi \sqrt{5 / 6}}\right)=\frac{1}{2} \sqrt[3]{1-3 D_{5 / 2}^{4}}$

Proof. To prove the above results, we need to apply Corollary 3 to Lemma 2(iv) respectively.

Theorem 12. We have
i. $\quad h_{2,3}=\sqrt{\sqrt{6}+\sqrt{3}-\sqrt{2}-2}=h_{2,1 / 3}^{-1}$,
ii. $\quad h_{2,1 / 9}=\sqrt{35-24 \sqrt{2}-2 \sqrt{6(99-70 \sqrt{2})}}=h_{2,9}^{-1}$.

Proof. On employing the definition of $h_{k, n}$ in Theorem 5 and setting $n=1 / 3$, we deduce

$$
x^{4}+\frac{1}{x^{4}}-36\left(x^{2}+\frac{1}{x^{2}}\right)+96 \sqrt{2}\left(x+\frac{1}{x}\right)+202=0 .
$$

where $x=h_{2,3}^{2}$. On setting $x+x^{-1}=t$, we obtain

$$
t^{4}-40 t^{2}+96 \sqrt{2} t-128=0 .
$$

On solving, we obtain $t=-2(\sqrt{6}+\sqrt{2}), 2(\sqrt{6}-\sqrt{2})$ and $2 \sqrt{2}$ as a double root. Since $h_{k, n}$ is a decreasing function, we choose

$$
x+\frac{1}{x}=2(\sqrt{6}-\sqrt{2}) .
$$

On solving this, we obtain the first identity. Similarly we obtain $h_{2,1 / 9}$ by setting $n=1 / 9$ in Theorem 5 .

Corollary 5. We have

$$
\begin{aligned}
\text { i. } & H\left(e^{-\pi \sqrt{3 / 2}}\right)=\sqrt{\frac{\sqrt[4]{2} h_{2,3}-1}{\sqrt[4]{2} h_{2,3}+1}}, & H\left(e^{-\pi / \sqrt{6}}\right)=\sqrt{\frac{\sqrt[4]{2}-h_{2,1 / 3}}{\sqrt[4]{2}+h_{2,1 / 3}}} \\
\text { ii. } & H\left(e^{-\pi / 3 \sqrt{2}}\right)=\sqrt{\frac{\sqrt[4]{2} h_{2,1 / 9}-1}{\sqrt[4]{2} h_{2,1 / 9}+1}}, & H\left(e^{-3 \pi / \sqrt{2}}\right)=\sqrt{\frac{\sqrt[4]{2}-h_{2,9}}{\sqrt[4]{2}+h_{2,9}}}
\end{aligned}
$$

Proof. The above results directly follows from Lemma 1 and Theorem 12.

Theorem 13. We have

$$
h_{3,4}=\frac{1}{2}(2-\sqrt{6}+\sqrt{14-4 \sqrt{6}})=h_{3,1 / 4}^{-1} .
$$

Proof. On using the definition of $h_{k, n}$ in Theorem 7 and setting $n=1 / 4$, we deduce

$$
x^{4}+\frac{1}{x^{4}}-4\left(x^{3}-\frac{1}{x^{3}}\right)-6\left(x^{2}+\frac{1}{x^{2}}\right)+12\left(x-\frac{1}{x}\right)+10=0,
$$


where $x=h_{3,4}^{2}$ and on setting $x+x^{-1}=t$, we have

$$
t^{2}\left(t^{2}-4 t-2\right)=0
$$

On solving, we obtain $t=0,2+\sqrt{6}$ and $2-\sqrt{6}$. Since $0<h_{k, n}<1$, we choose $x-x^{-1}=2-\sqrt{6}$. Further on solving this, we obtain $h_{3,4}$.

Corollary 6. We have

$$
D_{4}=\sqrt{\frac{4 \sqrt{2}+2 \sqrt{6}-2 \sqrt{3}-6}{6 \sqrt{3}-6 \sqrt{2}-4 \sqrt{6}+10}}, \quad D_{1 / 4}=\sqrt{\frac{6 \sqrt{3}-6 \sqrt{2}-4 \sqrt{6}+8}{4 \sqrt{3}-2 \sqrt{6}-4 \sqrt{2}+6}} .
$$

Proof. On using Theorem 13 to Lemma 2(iii), we obtain $D_{4}$ and $D_{1 / 4}$.

Corollary 7. We have

$$
G\left(e^{-2 \pi / \sqrt{3}}\right)=\frac{1}{2} \sqrt[3]{1-3 D_{4}^{4}}, \quad G\left(e^{-\pi / 2 \sqrt{3}}\right)=\frac{1}{2} \sqrt[3]{1-3 D_{1 / 4}^{4}}
$$

Proof. To prove the above results, we need to apply Corollary 6 to Lemma 2(iv) respectively.

Theorem 14. We have

$$
h_{3,7}=(2 \sqrt{7(7+4 \sqrt{3})}-6 \sqrt{3}-9)^{1 / 4}=h_{3,1 / 7}^{-1} .
$$

Proof. On using the definition of $h_{k, n}$ in Theorem 6 and setting $n=1 / 7$, we deduce

$$
\frac{1}{x^{4}}-x^{4}+14\left(\frac{1}{x^{2}}-x^{2}\right)-14 \sqrt{3}\left(\frac{1}{x^{2}}+x^{2}\right)+20 \sqrt{3}=0
$$

where $x=h_{3,7}^{2}$ and on solving we obtain the above result.

Corollary 8. We have

where

$$
D_{7}=\sqrt{\frac{\sqrt{3}-\sqrt{a}}{1+\sqrt{3 a}}}, \quad D_{1 / 7}=\sqrt{\frac{\sqrt{3} a-1}{a+\sqrt{3}}},
$$

$$
a=2 \sqrt{7(7+4 \sqrt{3})}-6 \sqrt{3}-9
$$

Proof. On using Theorem 14 to Lemma 2(iii), we obtain $D_{7}$ and $D_{1 / 7}$.

Corollary 9. We have

$$
G\left(e^{-\pi \sqrt{7 / 3}}\right)=\frac{1}{2} \sqrt[3]{1-3 D_{7}^{4}}, \quad G\left(e^{-\pi / \sqrt{21}}\right)=\frac{1}{2} \sqrt[3]{1-3 D_{1 / 7}^{4}}
$$

Proof. To prove the above results, we need to apply Corollary 8 to Lemma 2(iv) respectively. 
Theorem 15. We have
i. $\quad h_{3,2}=\sqrt{\sqrt{3}+\sqrt{8-4 \sqrt{3}}-2}=h_{3,1 / 2}^{-1}$,
ii. $\quad h_{3,12}=\sqrt{\frac{9+3 \sqrt[4]{3} \sqrt{2}-3 \sqrt{3}}{A+B}}=h_{3,1 / 12}^{-1}$,
iii. $\quad h_{3,4 / 3}=\sqrt{\frac{3-\sqrt{2} \sqrt[4]{3}+3 \sqrt{3}-2 D}{C}}=h_{3,3 / 4}^{-1}$,
iv. $\quad h_{3,20}=\sqrt{\frac{2 \sqrt{15}+2 \sqrt{3}+\sqrt{5}-9}{E}}=h_{3,1 / 20}^{-1}$,
v. $\quad h_{3,4 / 5}=\sqrt{\frac{\sqrt{3}-6 \sqrt{5}+\sqrt{15}+4 G-6}{F}}=h_{3,5 / 4}^{-1}$,
vi. $\quad h_{3,36}=\frac{1}{3}(H-I)=h_{3,1 / 36}^{-1}$,
vii. $\quad h_{3,4 / 9}=\frac{1}{3}(3-\sqrt{3}+\sqrt[3]{4} \sqrt{3}+J)=h_{3,9 / 4}^{-1}$,

where

$$
\begin{aligned}
A & =9 \sqrt{3}+6 \sqrt{2} \sqrt[4]{3}-3 \sqrt{2} \sqrt[4]{27}-9 \\
B & =\sqrt{3(96-120 \sqrt{2} \sqrt[4]{3}-48 \sqrt{3}+72 \sqrt{2} \sqrt[4]{27})} \\
C & =\sqrt{2} \sqrt[4]{27}+2 \sqrt{2} \sqrt[4]{3}-\sqrt{3}-3, \quad D=\sqrt{2(4-\sqrt{2} \sqrt[4]{3}+2 \sqrt{3}-\sqrt{2} \sqrt[4]{27})} \\
E & =6+\sqrt{3}-6 \sqrt{5}-\sqrt{15}-4 \sqrt{2(2+\sqrt{3})(3-\sqrt{5})} \\
F & =2 \sqrt{15}-3 \sqrt{5}+2 \sqrt{3}-11, \quad G=\sqrt{2(2-\sqrt{3})(3+\sqrt{5})} \\
H & =45+36 \sqrt[3]{2}+27 \sqrt[3]{4}+(25+20 \sqrt[3]{2}+16 \sqrt[3]{4}) \sqrt{3} \\
I & =\sqrt{11430+9072 \sqrt[3]{2}+7200 \sqrt[3]{4}+6597 \sqrt{3}+5238 \sqrt[3]{2} \sqrt{3}+4158 \sqrt[3]{4} \sqrt{3}} \\
J & =\sqrt{6+12 \sqrt[3]{2}-12 \sqrt[3]{4}-3 \sqrt{3}-6 \sqrt[3]{2} \sqrt{3}+6 \sqrt[3]{4} \sqrt{3}}
\end{aligned}
$$

Proof. The above results directly follows from Theorem 3 and the definition of $h_{k, n}$, where we set $n=1 / 2,1,3,1 / 3,5,1 / 5,9$ and $1 / 9$ respectively by making use of Lemma 3.

Corollary 10. We have

$$
\begin{aligned}
\text { i. } & D_{2}=\sqrt{\sqrt{2}-1}, & D_{1 / 2}=\sqrt{\frac{2-2 \sqrt{3}+\sqrt{3(8-4 \sqrt{3})}}{2 \sqrt{3}-2+\sqrt{8-4 \sqrt{3}}},} \\
\text { ii. } & D_{12}=\sqrt{\frac{\sqrt{3}-a^{2}}{1+\sqrt{3} a^{2}}}, & D_{1 / 12}=\sqrt{\frac{\sqrt{3} a^{2}-1}{a^{2}+\sqrt{3}}},
\end{aligned}
$$



iii. $\quad D_{4 / 3}=\sqrt{\frac{\sqrt{3}-b^{2}}{1+\sqrt{3} b^{2}}}$,
$D_{3 / 4}=\sqrt{\frac{\sqrt{3} b^{2}-1}{b^{2}+\sqrt{3}}}$,
iv. $\quad D_{20}=\sqrt{\frac{\sqrt{3}-c^{2}}{1+\sqrt{3} c^{2}}}$,
$D_{1 / 20}=\sqrt{\frac{\sqrt{3} c^{2}-1}{c^{2}+\sqrt{3}}}$,
v. $\quad D_{4 / 5}=\sqrt{\frac{\sqrt{3}-d^{2}}{1+\sqrt{3} d^{2}}}$,
$D_{5 / 4}=\sqrt{\frac{\sqrt{3} d^{2}-1}{d^{2}+\sqrt{3}}}$,
vi. $\quad D_{36}=\sqrt{\frac{\sqrt{3}-e^{2}}{1+\sqrt{3} e^{2}}}$,
$D_{1 / 36}=\sqrt{\frac{\sqrt{3} e^{2}-1}{e^{2}+\sqrt{3}}}$,
vii. $\quad D_{4 / 9}=\sqrt{\frac{\sqrt{3}-f^{2}}{1+\sqrt{3} f^{2}}}$,
$D_{9 / 4}=\sqrt{\frac{\sqrt{3} f^{2}-1}{f^{2}+\sqrt{3}}}$,

where

$$
\begin{aligned}
a & =\sqrt{\frac{9+3 \sqrt[4]{3} \sqrt{2}-3 \sqrt{3}}{A+B}}, & b & =\sqrt{\frac{3-\sqrt{2} \sqrt[4]{3}+3 \sqrt{3}-2 D}{C},} \\
c & =\sqrt{\frac{2 \sqrt{15}+2 \sqrt{3}+\sqrt{5}-9}{E}}, & d & =\sqrt{\frac{\sqrt{3}-6 \sqrt{5}+\sqrt{15}+4 G-6}{F},} \\
e & =\frac{1}{3}(H-I), & f & =\frac{1}{3}(3-\sqrt{3}+\sqrt[3]{4} \sqrt{3}+J) .
\end{aligned}
$$

Here A, B, $C, D, E, F, G, H$, Iand $J$ are as defined in Theorem 15 .

Proof. On using Theorem 15 to Lemma 2(iii), we obtain the above results.

Corollary 11. We have
i. $\quad G\left(e^{-\pi \sqrt{2 / 3}}\right)=\frac{1}{2} \sqrt[3]{36 \sqrt{2}-50}$,
$G\left(e^{-\pi / \sqrt{6}}\right)=\frac{1}{2} \sqrt[3]{1-3 D_{1 / 2}^{4}}$,
ii. $\quad G\left(e^{-2 \pi}\right)=\frac{1}{2} \sqrt[3]{1-3 D_{12}^{4}}$,
$G\left(e^{-\pi / 6}\right)=\frac{1}{2} \sqrt[3]{1-3 D_{1 / 12}^{4}}$,
iii. $\quad G\left(e^{-2 \pi / 3}\right)=\frac{1}{2} \sqrt[3]{1-3 D_{4 / 3}^{4}}$,
$G\left(e^{-\pi / 2}\right)=\frac{1}{2} \sqrt[3]{1-3 D_{3 / 4}^{4}}$,
iv. $\quad G\left(e^{-\pi \sqrt{20 / 3}}\right)=\frac{1}{2} \sqrt[3]{1-3 D_{20}^{4}}$,
$G\left(e^{-\pi 2 \sqrt{15}}\right)=\frac{1}{2} \sqrt[3]{1-3 D_{1 / 20}^{4}}$,
v. $\quad G\left(e^{-2 \pi / \sqrt{15}}\right)=\frac{1}{2} \sqrt[3]{1-3 D_{4 / 5}^{4}}$,
$G\left(e^{-\sqrt{5} \pi / 2 \sqrt{3}}\right)=\frac{1}{2} \sqrt[3]{1-3 D_{5 / 4}^{4}}$,
vi. $\quad G\left(e^{-2 \sqrt{3} \pi}\right)=\frac{1}{2} \sqrt[3]{1-3 D_{36}^{4}}$,
$G\left(e^{-\pi / 6 \sqrt{3}}\right)=\frac{1}{2} \sqrt[3]{1-3 D_{1 / 36}^{4}}$,
vii. $\quad G\left(e^{-2 \pi / 3 \sqrt{3}}\right)=\frac{1}{2} \sqrt[3]{1-3 D_{4 / 9}^{4}}$,
$G\left(e^{-\sqrt{3} \pi / 2}\right)=\frac{1}{2} \sqrt[3]{1-3 D_{9 / 4}^{4}}$.

Proof. To prove the above results, we need to apply Corollary 10 to Lemma 2(iv) respectively. 
Theorem 16. We have

$$
\begin{aligned}
& h_{3,1 / 3}^{\prime}=\sqrt{\frac{6 \sqrt{3}-\sqrt{2} \sqrt[4]{27}-3 \sqrt{2} \sqrt[4]{3}}{3(2+\sqrt{2} \sqrt[4]{3}+\sqrt{2} \sqrt[4]{27})}}, h_{3,5}^{\prime}=\sqrt{\frac{\sqrt{6(\sqrt{5}+1)}-\sqrt{2(\sqrt{5}-1)}}{\sqrt{2(\sqrt{5}+1)}+\sqrt{6(\sqrt{5}-1)}}}, \\
& h_{3,1 / 5}^{\prime}=\sqrt{\frac{\sqrt{6(\sqrt{5}-1)}-\sqrt{2(\sqrt{5}+1)}}{\sqrt{2(\sqrt{5}-1)}+\sqrt{6(\sqrt{5}+1)}}}, \quad h_{3,9}^{\prime}=\sqrt{\frac{\sqrt{3}-\sqrt[3]{4} \sqrt{3}+3}{\sqrt{3}+3 \sqrt[3]{4}-3}}, \\
& h_{3,1 / 9}^{\prime}=\sqrt{\frac{9-\sqrt{3}-2 \sqrt[3]{2} \sqrt{3}-\sqrt[3]{4} \sqrt{3}}{3(1+2 \sqrt[3]{2}+\sqrt[3]{4}+\sqrt{3})}} .
\end{aligned}
$$

Proof. The above results directly follows from Theorem 1 and the definition of $h_{k, n}$, where we set $n=1 / 3,5,1 / 5,9$ and $1 / 9$ respectively by making use of Lemma 3.

Theorem 17. We have

where

i. $\quad h_{2,16}=\frac{2}{\sqrt{2 \sqrt{2}+4 \sqrt{2((13 \sqrt{2}+18) \sqrt{1+\sqrt{2}}-20 \sqrt{2}-28)}}}=h_{2,1 / 16}^{-1}$,

ii. $\quad h_{2,32}=\sqrt{\frac{6+8 \sqrt[4]{2}+12 \sqrt{2}+8 \sqrt[4]{8}}{A}}=h_{2,1 / 32}^{-1}$,

$$
A=12+8 \sqrt[4]{2}+3 \sqrt{2}+4 \sqrt[4]{8}+\sqrt{256+240 \sqrt[4]{2}+192 \sqrt{2}+144 \sqrt[4]{8}}
$$

Proof. The above results directly follows from Theorem 4 and the definition of $h_{k, n}$, where we set $n=4$ and 16 respectively by making use of Lemma 3 .

Corollary 12. We have
i. $H\left(e^{-2 \sqrt{2} \pi}\right)=\sqrt{\frac{\sqrt[4]{2} h_{2,16}-1}{\sqrt[4]{2} h_{2,16}+1}}$
ii. $H\left(e^{-4 \pi}\right)=\sqrt{\frac{\sqrt[4]{2} h_{2,32}-1}{\sqrt[4]{2} h_{2,32}+1}}$,

$$
\begin{aligned}
& H\left(e^{-\pi / 4 \sqrt{2}}\right)=\sqrt{\frac{\sqrt[4]{2}-h_{2,1 / 16}}{\sqrt[4]{2}+h_{2,1 / 16}}}, \\
& H\left(e^{-\pi / 8}\right)=\sqrt{\frac{\sqrt[4]{2}-h_{2,1 / 32}}{\sqrt[4]{2}+h_{2,1 / 32}}}
\end{aligned}
$$

Proof. The above results directly follows from Lemma 1 and Theorem 17.

Theorem 18. We have

$$
h_{3,4}^{\prime}=\frac{\sqrt{3}-1}{\sqrt{2}}, \quad \text { and } \quad h_{3,1 / 4}^{\prime}=\left(\frac{3-2 \sqrt{2}-\sqrt{3}+\sqrt{6}}{3+\sqrt{2}-\sqrt{3}}\right)^{1 / 4} .
$$


Proof. The above results directly follows from Theorem 2 and the definition of $h_{k, n}$, where we set $n=1$ and $1 / 4$ respectively by making use of Lemma 3 .

\section{ACKNOWLEDGMENT}

The authors thank anonymous referee for valuable suggestions and comments.

\section{REFERENCES}

[1] C. Adiga, K. R. Vasuki, and M. S. M. Naika, "Some new explicit evaluations of ramanujan's cubic continued fractions," New Zealand Journal of Mathematics, vol. 31, pp. 109-117, 2002.

[2] C. Adiga, N. A. S. Bulkhali, D. Ranganatha, and H. M. Srivastava, "Some new modular relations for the rogers-ramanujan type of order eleven with applications to partitions," Journal of Number Theory, vol. 158, pp. 281-297, 2016, doi: 10.1016/j.jnt.2015.06.019.

[3] N. D. Baruah and N. Saikia, "Some general theorems on the explicit evaluations of ramanujan's cubic continued fraction," J. Comp. Appl. Math., vol. 160, pp. 37-51, 2003, doi: 10.1016/S03770427(03)00612-5.

[4] B. C. Berndt, Ramanujan's Notebooks Part III. New York: Springer, 1991.

[5] B. C. Berndt, Ramanujan's Notebooks Part V. New York: Springer, 1998.

[6] B. C. Berndt, H. H. Chan, and L. C. Zhang, "Ramanujan's class invariants and cubic continued fraction," Acta Arith., vol. 73, pp. 67-85, 1995, doi: 10.4064/aa-73-1-67-85.

[7] B. C. Berndt, H. H. Chan, and L. C. Zhang, "Ramanujan's explicit values for the classical thetafunction," Mathematika, vol. 42, pp. 278-294, 1995, doi: 10.1112/S0025579300014595.

[8] S. Bhargava, K. R. Vasuki, and T. G. Sreeramamurthy, "Some evaluations of ramanujan's cubic continued fraction," Indian Journal Pure Applied Math, vol. 35, no. 8, pp. 1003-1025, 2004.

[9] H. H. Chan, "On ramanujan's cubic continued fraction,” Acta Arith., vol. 73, pp. 343-345, 1995, doi: 10.4064/aa-73-4-343-355.

[10] H. H. Chan and S. S. Huang, "On the ramanujan-gollnitz-gordan continued fraction," The Ramanujan J., vol. 1, pp. 75-90, 1997.

[11] H. Gollnitz, "Partition mit differnzenbendingungen," J. Reine Angew. Math., vol. 25, pp. 154-190, 1967.

[12] B. Gordon, "Some continued fractions of the roger's ramanujan type," Duke Math. J., vol. 32, pp. 741-748, 1965, doi: 10.1215/S0012-7094-65-03278-3.

[13] M. S. M. Naika and S. Chandan Kumar, "Some new modular equations and their applications," Tamsui Oxford J. of Information and Mathematical sciences, vol. 28, no. 4, pp. 437-469, 2012.

[14] M. S. M. Naika, K. Sushan Bairy, and M. Manjunatha, "Some new modular equations of degree four and their explicit evaluations," European J. of pure and applied mathematics, vol. 3, no. 6, pp. 924-947, 2010.

[15] S. Ramanujan, The lost notebook and other unpublished papers. New Delhi: Narosa, 1957.

[16] S. Ramanujan, Notebooks (2 Volumes). Bombay: Tata Institute of Fundamental Research, 1957.

[17] H. M. Srivastava and M. P. Chaudhary, "Some relationships between $q$-product identities, combinatorial partition identities and continued-fractions identities," Adv. Stud. Contemp. Math., vol. 25, no. 3, pp. 265-272, 2015.

[18] H. M. Srivastava, M. P. Chaudhary, and S. Chaudhary, "Some theta function identities related to jacobi's triple product identity,” Eur. J. Pure Appl. Math., vol. 11, no. 1, pp. 1-9, 2018.

[19] H. M. Srivastava, M. P. Chaudhary, and S. Chaudhary, "A family of theta function identities related to jacobi's triple product identity," Russian J. of Math. Phys, vol. 27, no. 1, pp. 139-144, 2020. 
[20] B. R. Srivatsa Kumar, T. G. Sreeramamurthy, and G. N. Rajappa, "Evaluations of ramanujan's cubic continued fraction $\mathrm{g}(\mathrm{q})$ by modular equations," J. of Ramanujan Society of Math. and Math. Sc., vol. 1, no. 1, pp. 49-68, 2012.

[21] K. R. Vasuki and T. G. Sreeramamurthy, "A note on p-q modular equations," Tamsui Oxford J. of Mathematical Sciences, vol. 21, no. 2, pp. 109-120, 2005.

[22] K. R. Vasuki and B. R. Srivatsa Kumar, "Evaluations of the ramanujan-göllnitz-gordan continued fraction h(q) by modular equations," Indian J. of Mathematics, vol. 48, no. 3, pp. 275-300, 2006.

[23] E. T. Whittaker and G. N. Watson, A course of modern analysis. Cambridge: Cambridge University Press, 1966.

[24] J. Yi, "Theta-function identities and the explicit formulas for theta-function and their applications," J. Math. Anal. Appl., vol. 292, pp. 381-400, 2004, doi: 10.1016/j.jmaa.2003.12.009.

Authors' addresses

Shruthi

Department of Mathematics, Manipal Institute of Technology, Manipal Academy of Higher Education, Manipal - 576 104, India

E-mail address: shruthikarranthegmail.com

\section{B. R. Srivatsa Kumar}

(Corresponding author) Department of Mathematics, Manipal Institute of Technology, Manipal Academy of Higher Education, Manipal - 576 104, India,

E-mail address: sri_vatsabr@yahoo.com 